\title{
Onset of loneliness in older adults: results of a 28 year prospective study
}

\author{
Marja Aartsen • Marja Jylhä
}

Published online: 5 February 2011

(C) The Author(s) 2011. This article is published with open access at Springerlink.com

\begin{abstract}
The goal of this research is to test whether often observed correlates of loneliness in older age are related to onset of loneliness longitudinally. Despite the increasing number of longitudinal studies, the investigation of factors that are related to onset of loneliness is still limited. Analyses are based on data of the TamELSA study, which is a population-based prospective study in Tampere, Finland and started in 1979. For the present study 469 older adults aged between 60 and 86 years at baseline, who were not lonely at baseline, were selected and followed-up in 1989, 1999 and 2006. During the 28 years of follow-up approximately one third $(N=178)$ of the study population developed feelings of loneliness. Logistic regression analyses indicated that losing a partner, reduced social activities, increased physical disabilities, increased feelings of low mood, uselessness and nervousness, rather than baseline characteristics, are related to enhanced feelings of loneliness at follow-up. The higher incidence of loneliness among women can be fully explained by the unequal distribution of risk factors among men and women (e.g., women more often become widowed). Our results are in line with the cognitive approach that conceptualizes loneliness as an unpleasant feeling due to a perceived discrepancy between the desired and the achieved level of social and personal resources.
\end{abstract}

Communicated by Hans-Werner Wahl.

M. Aartsen · M. Jylhä

School of Public Health, University of Tampere,

Tampere, Finland

M. Aartsen $(\bowtie)$

VU University Amsterdam, Faculty of Social Sciences,

De Boelelaan, 1081 1081HV, Amsterdam

e-mail: m.j.aartsen@vu.nl
Keywords Correlates of loneliness - Older age

\section{Introduction}

Images of aging often include loneliness as a key characteristic of older age. However, the majority of scientific studies in this field indicate that the prevalence of severe loneliness (i.e., those who often feel lonely) among older people is relatively low, ranging from $3 \%$ in Nordic countries to approximately $30 \%$ in southern European countries (Jylhä and Jokela 1990). Yet, due to the adverse health consequences such as increased depression (Cacioppo et al. 2006) or increased likelihood of nursing home admissions (Russell et al. 1997), loneliness is nevertheless a relevant public health issue. Insight into factors that are associated with an increased risk of becoming lonely may reveal important information to our understanding of loneliness and to the design of interventions to prevent or reduce loneliness.

There is a growing body of knowledge about factors that are associated with loneliness in older age such as living alone (Wenger et al. 1996), quality of the relationship with children (Long and Martin 2000), socio economic status (Pinquart and Sörensen 2001), quality of the marriage (De Jong Gierveld et al. 2009), self-efficacy (Fry and Debats 2002), personality (Solano et al. 1982; Newall et al. 2009), cognition (Martin et al. 1997), self-perceived health (Kaasa 1998), and cultural norms and values (Jylhä and Jokela 1990) (see for a meta analysis Pinquart and Sörensen 2001). Also genetic factors have been found to increase the risk of loneliness (Boomsma et al. 2005). Results on gender differences in loneliness are still inconclusive, but as far as they have been detected they are generally small (De Jong Gierveld 1998, Dykstra and De Jong Gierveld 2004; Stokes 
and Levin 1986), and may be the consequence of the unequal distribution of risk factors across men and women (Pinquart and Sörensen 2001; Victor et al. 2005). Previous research thus revealed a wide range of factors, also referred to as the personal and social resources of the individual (De Jong Gierveld 1998).

Longitudinal studies on loneliness suggest that losing social and personal resources, for example increasing disability and decreasing social integration (Jylhä 2004), and the loss of a partner (Dykstra et al. 2005), lead to enhanced levels of loneliness. The acknowledgement of the importance of losing personal and social resources for the individual's feelings of loneliness shows similarities with ideas by Hobfoll $(1989,2001)$ who argues that social resources are important for well-being. People therefore strife to obtain and protect personal resources. Losing resources is disproportionately more influential on level of well-being than gaining resources (Hobfoll 2001).

The present study seeks to investigate the impact of baseline levels of a wide range of social and personal resources, and losses in these resources, on the onset of loneliness in older age. In accord with previous ideas and findings about loneliness we expect that negative changes (or losses) in resources increase the risk of becoming lonely at follow-up. Next to this, we will investigate to what extent gender differences can be explained in terms of a differential exposure to risk factors across men and women. The selection of resources included in this study is based on review studies by De Jong Gierveld (1998) and Pinquart and Sörensen (2001) and obviously on the availability of information in the dataset.

\section{Methods}

Data

Data were derived from the Tampere Longitudinal Study on Aging (TamELSA), which started as part of the World Health Organization's (WHO) Eleven Country Study on Health Care in 1979 (Heikkinen et al. 1983; Waters et al. 1989). The sample and procedures have been described in more detail elsewhere (Jylhä et al. 1992). In short, TamELSA is a longitudinal prospective study starting in 1979, with follow-up measures in 1989, 1999 and 2006. Individuals, aged between 60 and 89 years at baseline, were randomly selected from the population register of Tampere in Finland, stratified to gender and 5 year birth cohorts. The baseline sample consisted of 1,059 individuals of which $49.8 \%$ was male, representing 82 percent of the initially approached persons. In 1989, a second measurement round was conducted and 435 (of which $40.2 \%$ were men) of the baseline sample could be re-interviewed. The third measurement was conducted in 1999, among 123 survivors $(33.3 \%$ men) and the forth measurement was conducted in 2006 among 51 survivors $(27.5 \%$ men). In addition, a new sample $(N=395)$ of 208 men and 187 women aged between 60 and 69 years old, drawn from the Tampere population register was added to the study in 1989. In 1999, 275 (47.3\% men), and in 2006, 200 (45.0\% men) individuals could be re-interviewed.

Logistic regression with stepwise selection of predictors revealed that 28 years survival was related to younger age, better self rated health and female gender. Loneliness, level of education, and marital status were not related to survival. For the 18 year survival in the new cohort the same predictors were observed. In addition to younger age, better self rated health and female gender, also being married at baseline positively influenced survival. All interviews were conducted face-to-face at the homes of the respondents, except for the data collection in 2006, when interviews were conducted by telephone. Questions on the study variables were asked in the same way at all four waves.

\section{Study sample}

For the present study, a sub-sample $(N=469)$ of participants who were not lonely at baseline, and who had at least one follow-up measure was used. Data of both cohorts were merged by measurement cycle, not measurement year, and analyzed together. At first follow-up, 463 (96.5\%) of the 469 participants and at the second follow-up $304(42.5 \%)$ participants could be re-interviewed. At third follow-up 31 participants were re-interviewed $(6.5 \%)$. The large difference in participant numbers between the third and fourth measurement is mainly due to the later inclusion of the new cohort, for which only three measurement rounds were conducted.

\section{Measurements}

\section{Loneliness}

Loneliness is measured by a single question 'do you feel lonely' with response categories often, sometimes, never and don't know. For the analyses, the variable was recoded into three categories, i.e., never (1), sometimes (2) and often (3). People who answered the question about feelings of loneliness with "don't know" $(n=11)$ were removed from the analysis since we were not sure whether this answer indicated some loneliness, the presence of memory problems or other reasons. Onset of loneliness was defined to be present at the first, second or third follow-up if a 1 or 2 points increase (increase from not lonely to sometimes or often lonely) on the loneliness index occurred. Once the 
onset was defined, changes in loneliness in the subsequent follow-ups were not taken into account.

\section{Covariates}

Baseline measures and changes in partner status, having friends, being socially active, self rated health, physical disabilities, mood, nervousness, irritability and feelings of uselessness were included in the analytical models as potential risk factors for the onset of loneliness. Partner status was recoded into three categories; living with partner (1), never married, divorced or separated, hereafter referred to as living alone (2) and widowed (3). Having friends is measured with the question "Do you have any good friends" with answering categories yes (1) and no (2). Being socially active was based on the combination of five questions about participation during the past 12 months in the following activities; family ceremonies, going to the theatre, library visits, attending religious services, and traveling to a foreign country. Answering categories ranged from 0 (never) to 9 (9 times or more). Based on what has been done in an earlier study with this dataset (Jylhä 2004), a sum score of all activities was calculated with equal weights for all activities except family ceremonies, for which the score was halved. The respondent's social activity was rated as high (1) if the sum score of all activities was higher than 3 , moderate (2) if the sum score was 2 or 3 , and low (3) if the sum score was 0 or 1 . Selfperceived health was assessed with the question "How would you evaluate your present health" with answering categories fairly good to very good (1), average (2), and fairly bad to bad (3). Physical disabilities were based on 4 questions concerning basic physical activities of daily living (ADL) (able to using toilet, washing, dressing, and incontinence problems) and 2 questions concerning instrumental activities of daily living (iADL) (cooking and light household tasks). If no disabilities were reported on any of the activities, physical disability was evaluated as absent (1). If disabilities with the performance of at least one iADL, and one ADL were reported, physical disabilities were rated as moderate (2). Disabilities with at least two ADL functions were rated as severe physical disabilities (3).

The 2 week prevalence of low mood, irritability, and nervousness was assessed with the following questions; "In the last two weeks, have you suffered from low spirits or depression", “...irritability or bursts of anger", and "...nerves tensions or nervousness", respectively. Each question was recoded into three categories: no (1), sometimes (2), and often to nearly constantly (3). Feelings of uselessness were assessed with the question "Do you feel unnecessary?" with answering categories never (1), sometimes (2), and often (3). Irritability, nervousness and feelings of uselessness were only measured in 1979, 1989 and 1999 so participants of the 1979 cohort can have a maximum of three observations and participants of the 1989 cohort a maximum of two.

\section{Statistical analysis}

The analyses were conducted in several subsequent steps. After descriptive analyses on the characteristics of the participants, a bivariate logistic regression was conducted of loneliness onset (yes/no) on each baseline and change score of the risk factors, while controlling for age. Subsequently, the unique contribution to the onset of loneliness of each significant risk factor derived from the bivariate models was estimated in a multivariate logistic regression model. Since the time period between the measurement waves was rather extended, the direction of causation may still be questionable. Therefore, we finally conducted a logistic regression analysis of onset of loneliness after 18 or 20 years, regressed on changes in risk factors during the first 10 years.

\section{Results}

\section{Descriptive statistics}

In the first 10 years following after the first measurement round, 135 of the 469 (28.1\%) participants became lonely; 18 to 20 years after the first measurement another 37 participants became lonely and during the last 20 to 28 years again 6 participants became lonely, leading to a 28 years incidence rate of loneliness of $37.1 \%(n=178)$. Recovery from loneliness after people became lonely occurred in 28 cases (5.8\% of the study sample). Since this was not the topic of our study, we did not further analyze predictors of recovery. The basic characteristics of the participants and changes in risk factors are presented in Table 1. Though the selection of a subsample of older adults who were not lonely at baseline and who had at least one follow-up measure inevitably led to a selection of healthier and younger people, there was sufficient onset of loneliness and sufficient variability in baseline and change scores to serve our research aims.

\section{Risk factors of loneliness}

The significance of the risk factors was first analyzed in a bivariate logistic regression model, while controlling for the effect of age. Table 2 shows that, apart from gender, none of the baseline levels of the selected factors predicted onset of loneliness, unless the levels of these factors changed during follow-up. Women had a $48 \%$ higher 
Table 1 Baseline characteristics and changes in characteristics of the study sample $(N=469)$

\begin{tabular}{|c|c|c|}
\hline & $M, N$ & $\mathrm{SD}, \%$ \\
\hline Age $(M, \mathrm{SD})$ & 66.2 & 5.6 \\
\hline Female gender $(N, \%)$ & 256 & 53.3 \\
\hline \multicolumn{3}{|l|}{ Partner status $(N, \%)$} \\
\hline Living with partner & 325 & 67.7 \\
\hline No partner & 74 & 15.4 \\
\hline Widowed & 81 & 16.9 \\
\hline Loss of partner at FU $(N, \%)$ & 121 & 25.2 \\
\hline Having no friends $(N, \%)$ & 39 & 8.2 \\
\hline Loss of friends at $\mathrm{FU}(N, \%)$ & 67 & 14.0 \\
\hline \multicolumn{3}{|l|}{ Social activity $(N, \%)$} \\
\hline High activity & 367 & 77.3 \\
\hline Moderate & 42 & 8.8 \\
\hline Low & 66 & 13.9 \\
\hline Reduced social activity at FU $(N, \%)$ & 169 & 35.2 \\
\hline \multicolumn{3}{|l|}{ Self-perceived health $(N, \%)$} \\
\hline Good & 243 & 50.7 \\
\hline Average & 164 & 34.2 \\
\hline Poor & 72 & 15.0 \\
\hline Decline in self-perceived health at FU $(N, \%)$ & 137 & 28.5 \\
\hline \multicolumn{3}{|l|}{ Physical disabilities $(N, \%)$} \\
\hline Absent & 401 & 83.7 \\
\hline Moderate & 31 & 6.5 \\
\hline Severe & 47 & 9.8 \\
\hline Increased physical disabilities at FU $(N, \%)$ & 213 & 44.4 \\
\hline \multicolumn{3}{|l|}{ Feelings of low mood $(N, \%)$} \\
\hline Never & 402 & 84.5 \\
\hline Sometimes & 55 & 11.6 \\
\hline Often & 19 & 4.0 \\
\hline Increased feelings of low mood at FU $(N, \%)$ & 133 & 27.7 \\
\hline \multicolumn{3}{|l|}{ Feelings of irritation $(N, \%)$} \\
\hline Never & 395 & 83.2 \\
\hline Sometimes & 73 & 15.4 \\
\hline Often & 7 & 1.5 \\
\hline Increased feelings of irritations at $\mathrm{FU}(N, \%)$ & 63 & 13.1 \\
\hline \multicolumn{3}{|l|}{ Feelings of nervousness $(N, \%)$} \\
\hline Never & 360 & 75.5 \\
\hline Sometimes & 92 & 19.3 \\
\hline Often & 25 & 5.2 \\
\hline Increased feelings of nervousness at FU $(N, \%)$ & 77 & 16.0 \\
\hline \multicolumn{3}{|l|}{ Self esteem $(N, \%)$} \\
\hline Good & 412 & 88.6 \\
\hline Somewhat low & 49 & 10.5 \\
\hline Low & 4 & 0.9 \\
\hline Lowered self esteem at $\mathrm{FU}(N, \%)$ & 99 & 20.6 \\
\hline
\end{tabular}

probability to become lonely than men. Losing a partner increased the risk of becoming lonely with a factor 2.7 . People whose social activity level reduced were 2 times more likely to become lonely, and an increase in physical disabilities but not decrease in self-perceived physical health doubled the risks of becoming lonely. Increased feelings of low mood, nervousness, and uselessness led, respectively, to a 5.1, 3.3, and 4.5 times increased risk of becoming lonely.

To determine the unique contribution of the predictors found to be significant in the bivariate models, we conducted a stepwise multivariate logistic regression analysis with forward selection of the risk factors. Several significant bivariate associations between the factors and onset of loneliness lost their significance when other variables were taken into account. Variables that were no longer associated with onset of loneliness were gender, loss of friends, and loss of physical health (see Table 3). Risk factors remaining to have a unique contribution to the onset of loneliness are losing a partner, reduced social activities, increased feelings of low mood, nervousness and uselessness. The increase in the risk of becoming lonely varied from a factor 1.8 (reduction in social activities) to 4.0 (increased feelings of low mood). Taken together, these factors accounted for almost $30 \%$ of the variability in loneliness onset. Inspection of the collinearity statistics revealed that there was no collinearity among the risk factors (Correlations (Kendall's Tau) ranged from 0.05 to 0.21; Tolerance 0.89-0.94, VIF 1.06-1.13).

Since low mood, increased feelings of nervousness and increased feelings of uselessness are conceptually related constructs (Russell et al. 1980) we further investigated the zero-order correlates between these constructs and loneliness, and calculated which part of the explained variance in onset of loneliness could be accounted to these factors. The zero-order correlations between mood, irritability and uselessness reveals that correlations ranged from 0.10 for loneliness and irritability, to 0.42 for loneliness and feelings of uselessness suggesting that the overlap between the constructs and loneliness is weak to moderate. In addition, stepwise logistic regression with variables entered in three blocks revealed that age (first block), and gender, loss of the partner, loss of friends, loss of social activities, and reduced physical abilities (second block) could explain $13.4 \%$ of the total variance in onset of loneliness. An additional $16.6 \%$ of the total variance in loneliness was accounted to lowered mood, increased feelings of nervousness, and increased feelings of uselessness.

Since the time period between the measurement waves is rather extended, and loneliness and risk factors may have influenced each other mutually during the period between the measurements, we finally checked whether onset of loneliness between the second and third measurement could be predicted by changes in risk factors during the first 10 years (Table 4). As there were only 37 new loneliness cases between the second and third measurement, the 
Table 2 Bivariate associations between risk factors and onset of loneliness, controlling for age (reference group is not lonely)

\begin{tabular}{|c|c|c|c|c|c|}
\hline \multirow[t]{2}{*}{ Risk factors } & \multicolumn{5}{|c|}{ Lonely $(n=178 / 469)$} \\
\hline & OR & $95 \%$ C.I. & & $p$ & Wald statistic \\
\hline Age (per year increase) & 0.99 & 0.96 & 1.02 & 0.55 & 0.37 \\
\hline Being female & 1.59 & 1.08 & 2.33 & 0.02 & 5.50 \\
\hline \multicolumn{6}{|l|}{ Partner status } \\
\hline \multicolumn{6}{|l|}{ Living with partner is ref group } \\
\hline No partner & 1.03 & 0.61 & 1.74 & 0.92 & 0.01 \\
\hline Widowed & 1.00 & 0.51 & 1.96 & 1.00 & 0.00 \\
\hline Loss of partner at FU & 2.97 & 1.94 & 4.55 & $<0.001$ & 24.85 \\
\hline No friends & 1.02 & 0.50 & 2.06 & 0.97 & 0.00 \\
\hline Loss of friends at FU & 2.25 & 1.32 & 3.82 & 0.00 & 8.92 \\
\hline \multicolumn{6}{|l|}{ Social activity } \\
\hline \multicolumn{6}{|l|}{ High activity is ref group } \\
\hline Moderate & 0.91 & 0.52 & 1.58 & 0.73 & 0.12 \\
\hline Low & 0.97 & 0.42 & 2.22 & 0.95 & 0.01 \\
\hline Reduced social activity at FU & 2.08 & 1.40 & 3.08 & $<0.001$ & 13.03 \\
\hline \multicolumn{6}{|l|}{ Self-perceived health (SPH) } \\
\hline \multicolumn{6}{|l|}{ Good health is ref group } \\
\hline Average & 1.15 & 0.66 & 2.00 & 0.63 & 0.24 \\
\hline Poor & 0.94 & 0.53 & 1.68 & 0.84 & 0.04 \\
\hline Decline in $\mathrm{SPH}$ at $\mathrm{FU}$ & 1.36 & 0.90 & 2.06 & 0.14 & 2.14 \\
\hline \multicolumn{6}{|l|}{ Physical health } \\
\hline \multicolumn{6}{|l|}{ Good physical health is ref group } \\
\hline Average & 1.46 & 0.79 & 2.70 & 0.23 & 1.46 \\
\hline Poor & 1.90 & 0.72 & 5.03 & 0.19 & 1.69 \\
\hline Reduced physical health at FU & 2.19 & 1.49 & 3.21 & $<0.001$ & 15.84 \\
\hline \multicolumn{6}{|l|}{ Feelings of low mood } \\
\hline \multicolumn{6}{|l|}{ Never feelings of low mood is ref group } \\
\hline Sometimes & 1.57 & 0.60 & 4.09 & 0.35 & 0.86 \\
\hline Often & 1.08 & 0.37 & 3.18 & 0.89 & 0.02 \\
\hline Increased feelings of low mood at FU & 5.17 & 3.35 & 7.98 & $<0.001$ & 54.90 \\
\hline \multicolumn{6}{|l|}{ Feelings of irritation } \\
\hline \multicolumn{6}{|l|}{ Never feelings of irritation is ref group } \\
\hline Sometimes & 0.78 & 0.15 & 4.08 & 0.77 & 0.09 \\
\hline Often & 0.47 & 0.09 & 2.61 & 0.39 & 0.74 \\
\hline Increased feelings of irritations at FU & 1.29 & 0.75 & 2.24 & 0.36 & 0.83 \\
\hline \multicolumn{6}{|l|}{ Feelings of nervousness } \\
\hline \multicolumn{6}{|l|}{ Never feelings of nervousness is ref group } \\
\hline Sometimes & 1.26 & 0.54 & 2.95 & 0.59 & 0.29 \\
\hline Often & 0.65 & 0.26 & 1.64 & 0.36 & 0.84 \\
\hline Increased feelings of nervousness at FU & 3.24 & 1.94 & 5.40 & $<0.001$ & 20.26 \\
\hline \multicolumn{6}{|l|}{ Feelings of uselessness } \\
\hline \multicolumn{6}{|l|}{ Never feeling useless is ref group } \\
\hline Sometimes & 1.92 & 0.27 & 13.81 & 0.52 & 0.42 \\
\hline Often & 1.11 & 0.14 & 8.56 & 0.92 & 0.01 \\
\hline Increased feelings of uselessness at FU & 4.70 & 2.92 & 7.55 & $<0.001$ & 40.82 \\
\hline
\end{tabular}

Statistically significant $(p<0.05)$ odds ratios are displayed in bold 
Table 3 Final model for the multivariate associations between risk factors and onset of loneliness, controlling for age (reference group is not lonely, Method $=$ Forward stepwise selection of variables)

Nagelkerke $R^{2}=0.30$

Statistically significant $(p<0.05)$ odds ratios are displayed in bold

Table 4 Multivariate associations between changes in risk factors (T1-T2) and incidence of loneliness (T2-T3) controlling for age (reference group is no incident loneliness between $\mathrm{T} 1$ and $\mathrm{T} 2$, Method $=$ Enter)

\begin{tabular}{lllllc}
\hline Risk factors & \multicolumn{3}{l}{ Lonely $(n=178 / 469)$} & & \\
\cline { 2 - 6 } & OR & $95 \%$ C.I. & & \multicolumn{1}{c}{ Wald statistic } \\
\hline Age (per year increase) & 0.98 & 0.94 & 1.02 & 0.34 & 0.92 \\
Loss of partner at FU & $\mathbf{2 . 0 7}$ & 1.28 & 3.35 & 0.00 & 8.82 \\
Reduced social activities at FU & $\mathbf{1 . 8 0}$ & 1.15 & 2.83 & 0.01 & \multicolumn{1}{c}{6.56} \\
Increased feelings of low mood at FU & $\mathbf{4 . 0 4}$ & 2.53 & 6.44 & $<0.01$ & 34.33 \\
Increased feelings of nervousness at FU & $\mathbf{1 . 9 6}$ & 1.09 & 3.50 & 0.02 & 5.08 \\
Increased feelings of uselessness at FU & $\mathbf{3 . 5 1}$ & 2.08 & 5.91 & $<0.01$ & 22.30 \\
\hline
\end{tabular}

\begin{tabular}{lllllll}
\hline Risk factors & \multicolumn{7}{l}{ Lonely $(n=37 / 432)$} & & \\
\cline { 2 - 6 } & OR & $95 \%$ & C.I. & & $p$ & Wald statistic \\
\hline Age (per year increase) & 1.09 & 1.01 & 1.18 & 0.03 & 4.78 \\
Gender & 1.22 & 0.61 & 2.46 & 0.58 & 0.31 \\
Loss of partner between T1 and T2 & 1.56 & 0.52 & 4.65 & 0.42 & 0.64 \\
Loss of friends between T1 and T2 & 1.03 & 0.29 & 3.69 & 0.97 & 0.00 \\
Loss of social activities between T1 and T2 & 1.14 & 0.46 & 2.83 & 0.78 & 0.08 \\
Loss of physical health between T1 and T2 & 0.80 & 0.36 & 1.75 & 0.57 & 0.32 \\
Increased feelings of low mood between T1 and T2 & 1.26 & 0.49 & 3.27 & 0.63 & 0.23 \\
Increased feelings of nervousness between T1 and T2 & 1.70 & 0.24 & 1.47 & 0.25 & 1.30 \\
Increased feelings of uselessness between T1 and T2 & 1.52 & 0.50 & 4.66 & 0.47 & 0.53 \\
\hline
\end{tabular}

power to detect significance dropped considerably, so we only checked whether the directions were in accord with our results described above. Age and changes in risk factors in the first 10 years increased the risk of becoming lonely during the following 10 years, which reiterated our previous findings. In addition, these results suggest that changes in risk factors may have long lasting effects on the onset of loneliness.

\section{Discussion}

The aim of this study was to give more insight into the origins of loneliness in older adults by investigating whether changes in factors found to be associated with loneliness also leads to an enhanced likelihood of becoming lonely at follow-up. In addition, we investigated to what extent gender differences could be explained in terms of a differential exposure to risk factors across men and women. The study indicated that onset of loneliness in older age is more likely if people become more depressed, have increased feelings of uselessness, have increased feelings of nervousness, lose their partner, and have reduced levels of social activity. Baseline levels of social and psychological resources did not predict onset of loneliness. As far as men and women differ in feelings of loneliness, this can be explained in terms of a higher exposure to risk factors for women, and not to individual differences in vulnerability to loneliness between men and women.

Our findings are important in several ways. Firstly, this longitudinal study gave further support to the notion that losses in social and personal resources, rather than a small network or ill health, enhances the likelihood of becoming lonely. This fits to the idea that loneliness is understood as being the result of a subjective evaluation of the discrepancy between achieved, or rather the lost relationships, as compared with desired relationships.

Secondly, the study suggests that also losses in psychological resources, such as worsening of mental health, increase the likelihood of becoming lonely. More specifically, we found that increased feelings of uselessness, increased feelings of nervousness and increased feelings of low mood lead to a higher likelihood to become lonely in the future This is in line with the study of Newall et al. (2009) who found that control beliefs are important predictors of loneliness, and consistent with the theory of conservation of resources (Hobfoll 1988) stating that resources helping to maintain well-being can be social conditions (such as having a partner) but also psychological. It is also in line with the idea that personal characteristics can create difficulties for the person trying to establish or maintain satisfactory relationships (Perlman and Peplau 1981). In accord with the study of Fry and Debats (2002) we found that reductions in personal 
resources are even stronger predictors of loneliness than reductions in social resources.

One could argue that low mood, irritability, nervousness or feelings of uselessness are concepts that are closely related to loneliness, and may be considered two sides of the same coin. However, based on the zero-order correlations between mood, irritability and uselessness we may conclude that there is indeed overlap, but correlations are weak to moderate. In addition, the combined effect of mood, uselessness, irritability and nervousness only accounts for $17 \%$ of the variance in loneliness onset, leaving a substantive amount of variance to be explained by other factors.

Finally, it appears that a reduction in social activities leads to enhanced feelings of loneliness. With refer to the activity theory (Havighurst 1963) and the continuity theory (Atchley 1989) this increased feeling of loneliness may be the result of peoples inability to substitute activities or roles that are lost by new ones, which results in reduced wellbeing to which loneliness is closely related (e.g., Fees et al. 1999)

Our main finding that losses in social and personal resources, rather than their baseline level lead to a higher likelihood of becoming lonely may also indicate the existence of subgroups of people who differ in sensitivity to risk factors leading to different trajectories of loneliness development. In this study, we selected people who were not lonely at baseline. Given the long lasting effect of many of the risk factors observed in our study, it is striking that baseline levels did not predict loneliness. Since we started with a selection of people who were not lonely at baseline, we may have selected not only people that were fortunate enough to have not encountered any risk leading to enhanced levels of loneliness, but also people who have encountered risk events without affecting them. The acknowledgement of different subgroups is important for the theoretical and conceptual understanding of loneliness as well as for the development of intervention strategies (Victor et al. 2004). Further research on loneliness may benefit from insights into loneliness trajectories by identifying distinctive clusters of individual trajectories within the population, and see if covariates shaping the trajectories may have differential effect across the sub groups of people.

Several limitations of our study need to be acknowledged. There is a large time period between the follow-up measures, which may be problematic in causality research. The right time interval for causality to show up is highly dependent on the dynamics of the relationships between loneliness and its correlates. For example, Guiaux and colleagues showed that support from members in the personal network of widowed people, starts to increase already before widowhood, continues shortly after widowhood, but than returns to lower levels in the long run (Guiaux et al. 2007). In addition, onset of loneliness at the first follow-up could have occurred shortly after the baseline measure and we can not rule out the possibility that onset of loneliness might have led to lowered levels of mood or increased feelings of irritability. Nevertheless, our additional analyses testing whether onset of loneliness between the second and third measurement could be predicted by changes in risk factors during the first 10 years were in line with our results, which made us feel comfortable with our general conclusions.

Another limitation of our long follow-up period is the large attrition of the study sample, which has resulted in a healthier, and younger study sample compared to the original population. Next, change scores are based on observed data, which may have lead to some overestimation of change due to measurement error (Bassi et al. 2000). An alternative approach in this study could have been the use of Latent Growth Models to account for missing values, overestimation of change, the potential correlations between error terms, and the unreliability of measurements. Two potentially interesting attributes of growth trajectories are rates of change and initial status (Duncan and Duncan 2004). In these models, the time perspective is an important feature as changes are modeled over time. However, in this specific instance we preferred less emphasis on the influence of time by using clear-cut categories of the selected variables such as onset of loneliness versus no onset and loss of resources versus no loss of resources.

Finally, some of the selected study variables especially the psychological factors mood, irritability, and nervousness were assessed with a limited number of items and a limited number of answering categories. Therefore, reliability and the validity of these concepts may be lower than in well-established measurement scales or clinical assessments. It may be of interest to see if the effect of changes in personality factors is sustained if more elaborated questionnaires or clinical criteria are used.

An advantage of the longitudinal design is clearly the possibility to investigate the potential causal effect of (changes) in resources on the likelihood of becoming lonely in the future. Despite the long follow-up and the large attrition, our study sheds new light on the relative importance of social and psychological resources in preventing older people from becoming lonely. The study allowed us to conclude that some, but not all, correlates of loneliness generally believed to enhance loneliness are also related to an increased likelihood of becoming lonely. In particular, losses of social and psychological resources rather than baseline characteristics are related to an increased likelihood of becoming lonely. Gender differences in loneliness seem to be the result of the unequal 
exposure to risk factors (e.g., women become more often widowed).

Acknowledgment The Tampere Longitudinal Study in Ageing as been financed by the Academy of Finland and the Ministry of Social Affairs and health as part of the Research Programme on Ageing.

Open Access This article is distributed under the terms of the Creative Commons Attribution Noncommercial License which permits any noncommercial use, distribution, and reproduction in any medium, provided the original author(s) and source are credited.

\section{References}

Atchley R (1989) A continuity theory of normal aging. The Gerontologist 29:183-190

Bassi F, Hagenaars JA, Croon MA, Vermunt JK (2000) Estimating true changes when categorical panel data are affected by uncorrelated and correlated classification errors: an application to unemployment data. Sociol Methods Res 29:230-268

Boomsma DI, Willemsen G, Dolan CV, Hawkley LC, Cacioppo JT (2005) Genetic and environmental contributions to loneliness in adults: the Netherlands twin register study 35:745-752

Cacioppo JT, Hughes ME, Waite LJ, Hawkley LC, Thisted RH (2006) Loneliness as a specific risk factor for depressive symptoms: cross-sectional and longitudinal analyses. Psychol Aging 21:140-151

De Jong Gierveld J (1998) A review of loneliness: concept and definitions, determinants and consequences. Rev Clin Gerontol 8:73-80

De Jong Gierveld J, Broese van Groenou M, Hoogendoorn AW, Smit JH (2009) Quality of marriages in later life and emotional and social loneliness. J Gerontol 64B:S497-S506

Duncan TE, Duncan SC (2004) An introduction to latent growth modeling. Behav Ther 35:333-363

Dykstra PA, de Jong Gierveld J (2004) Gender and marital-history differences in emotional and social loneliness among Dutch older adults. Can J Aging 23:141-155

Dykstra PA, van Tilburg TG, de Jong Gierveld J (2005) Changes in older adult loneliness: results from a seven-year longitudinal study. Res Aging 27:725-747

Fees BS, Martin P, Poon LW (1999) A model of loneliness in older adults. J Gerontol Psychol Sci 54B:P231-P239

Fry PS, Debats DL (2002) Self-efficacy beliefs as predictors of loneliness and psychological distress in older adults. Int J Hum Dev 55:233-269

Guiaux M, van Tilburg TG, Broese van Groenou MB (2007) Changes in contact and support exchange in personal networks after widowhood. Pers Relationsh 14:457-473

Havighurst RJ (1963) Successful aging. In: Williams RH, Tibbitts C, Donahue W (eds) Processes of aging, vol 1. Atherton, New York

Heikkinen E, Waters WE, Brzezinski ZJ (1983) The elderly in eleven countries: a social-medical survey. World health organization, regional office for Europe (Public Health in Europe 21), Copenhagen, Denmark
Hobfoll SE (1988) The ecology of stress. Hemisphere, Washington, DC

Hobfoll SE (1989) Conservation of resources: a new attempt at conceptualizing stress. Am Psychol 44:513-524

Hobfoll SE (2001) The influence of culture, community, and the nested-self in the stress process: advancing conservation of resources theory. Appl Psychol 50:337-421

Jylhä M (2004) Old age and loneliness: cross-sectional and longitudinal analyses in the Tampere longitudinal study on aging. Can J Aging 23:157-168

Jylhä M, Jokela J (1990) Individual experiences as cultural-a crosscultural study on loneliness among the elderly. Ageing Soc 10:295-315

Jylhä M, Jokela J, Tolvanen E, Heikkinen E, Heikkinen RL, Koskinen S, Leskinen E, Lyyra AL, Pohjolainen P (1992) The Tampere longitudinal study on aging. Description of the study, basic results on health and functional ability. Scand J Soc Med 47: $1-58$

Kaasa KRN (1998) Loneliness in old age: psychosocial and health predictors. Nor J Epidemiol 8:195-201

Long MV, Martin P (2000) Personality, relationship closeness, and loneliness of oldest old adults and their children. J Gerontol 55B:P311-P319

Martin P, Hagberg B, Poon LW (1997) Predictors of loneliness in centenarians: a parallel study. J Cross-Cult Gerontol 12:203-224

Newall NE, Chipperfield JG, Clifton RA, Perry RP, Swift AU (2009) Causal beliefs, social participation, and loneliness among older adults: a longitudinal study. J Soc Pers Relationsh 26:273-290

Perlman D, Peplau LA (1981) Toward a social psychology of loneliness. In: Gilmour R, Duck S (eds) Personal relationships in disorder. Academic Press, London, pp 31-56

Pinquart M, Sörensen S (2001) Influences on loneliness in older adults: a meta-analysis. Basic Appl Soc Psychol 23:245-266

Russell D, Peplau LA, Cutrona CE (1980) The revised UCLA loneliness scale: concurrent and discriminant validity evidence. J Pers Soc Psychol 39:472-480

Russell DW, Cutrona CE, de la Mora A, Wallace RB (1997) Loneliness and nursing home admission among rural older adults. Psychol Aging 12:574-589

Solano CH, Batten PG, Parish EA (1982) Loneliness and patterns of self-disclosure. J Pers Soc Psychol 43:524-531

Stokes J, Levin I (1986) Gender differences in predicting loneliness from social network characteristics. J Pers Soc Psychol 51: 1069-1074

Victor CR, Scambler SJ, Bond J, Bowling A (2004) Loneliness in later life. In: Walker A, Hennesey C (eds) Quality of life in old age. Open University press, Maidenhead, Birkshire

Victor CR, Scambler SJ, Marston L, Bond J, Bowling A (2005) Older people's experiences of loneliness in the UK: does gender matter? Soc Policy Soc 5:27-38

Waters WE, Heikkinen E, Dontas AS (1989) Health lifestyles and services for the elderly. World health organization, regional office for Europe (Public Health in Europe 21), Copenhagen Denmark

Wenger GC, Davies R, Shahtahmasebi S, Scott A (1996) Social isolation and loneliness in old age: review and model refinement. Ageing Soc 16:333-358 